\title{
Ernährungstherapie und therapeutisches Fasten in der Naturheilkunde
}

\author{
Andreas Michalsen \\ Kliniken Essen-Mitte, Abteilung Innere Medizin V, Integrative Medizin und Naturheilkunde, DE-Essen
}

n der Systematik der sog. klassischen Naturheilverfahren bilden, entsprechend der Definition der Europäischen Gesellschaft für Komplementärmedizin und Naturheilkunde (ESCNM), Ernährungstherapie und Fastentherapie, neben Ordnungstherapie, Physikalischen Therapien inklusive Hydro-, Balneo-, Klima und Bewegungstherapie und Phytotherapie, die zentralen Therapieverfahren. Mit dem Aufkommen industrialisierter Lebens- und Gesellschaftsformen formulierte die $\mathrm{Na}$ turheilbewegung im 19. Jahrhundert das Primat einer naturgemäfen Lebensform und einer naturgemässen Diät als grundlegende Massnahme jeder medizinischen Therapie [1]. In diesem Zusammenhang und im Rückgriff auf die klassische hippokratische Diätetik wurde von wesentlichen Protagonisten der Naturheilkunde wie Max Bircher-Benner und später Werner Kollath und Helmut Anemüller das Konzept einer „vollwertigen“ Ernährung entwickelt und konzipiert. BIRCHER-BENNER therapierte Patienten mit einem Schwerpunkt auf pflanzliche Frischkost, der er einen spezifischen Energiegehalt gegenüber allen verarbeiteten, stark erhitzten und tierischen Nahrungsmitteln zusprach [2]. WERNER KOLLATH führte später das Konzept der Vollwertkost ein. Grundgedanke war das Postulat, Lebensmittel seien umso wertvoller und gesünder, je weniger sie bearbeitet werden. KoLLATH unterteilte die Lebensmittel entsprechend in Wertstufen [3]. ANEMÜLLER schliesslich entwickelte das nach ihm benannte Grunddiätsystem als ein Ernährungskonzept sowohl für die Prävention als auch für die klinische Diätetik [4]. Diese Grundkonzepte
Hintergrund: Umfangreiche Studienergebnisse der letzten Jahre weisen der Ernährungstherapie eine vorrangige Bedeutung in der Prävention und Therapie chronischer Erkrankungen zu. Grundlagenwissenschaftlich wird zunehmend die gesundheitsfördernde Wirkung kalorischer Restriktion und des intermittierenden Fastens diskutiert. Umfassende Konzepte einer intensivierten Ernährungstherapie unter Einbindung der Fastentherapie sind traditioneller Bestandteil naturheilkundlicher Behandlungsansätze. Zielsetzung: Übersicht über die aus dem bisherigen Kenntnisstand abzuleitenden Therapieempfehlungen in der Ernährungsmedizin und Positionierung der naturheilkundlichen Ernährungsgrundsätze und des therapeutischen Fastens im integrativen Therapieverständnis. Methoden: Analyse und Bewertung von Humanstudien (randomisierte Studien, epidemiologische Studien, experimentell-deskriptive Studien), von tierexperimentellen Studien sowie der Angaben von publizierten Standardwerken. Ergebnisse und Schlussfolgerungen: Traditionelle Ernährungsformen, wie insbesondere die mediterrane Ernährung, sind ein hoch wirksames Therapieprinzip in der Behandlung der koronaren Herzerkrankung, des metabolischen Syndroms, des Diabetes mellitus und der rheumatoiden Arthritis. Die in der Naturheilkunde entwickelte Vollwerternährung mit hohen Frischkostanteilen unverarbeiteter Lebensmittel zeigt eine Vielzahl gesundheitsfördernder Effekte. Die kombinierte Mediterrane Vollwerternährung erweist sich in der klinischen Anwendung als gut umsetzbar und klinisch vielversprechend. Das therapeutische modifizierte Fasten ist eine wirksame und sichere Therapiemethode in der Behandlung der rheumatoiden Arthritis und des Bluthochdrucks und unterstützt Lebensstilmodifikation und Ernährungsumstellung. Ergebnisse aus Beobachtungsstudien weisen auf einen potentiellen Nutzen bei chronischen Schmerzsyndromen, entzündlichen und kardiovaskulären Erkrankungen. Experimentelle Daten belegen ausgeprägte Effekte des intermittierenden Fasten auf degenerative und altersassoziierte zelluläre Prozesse. Das Potential des therapeutischen Fastens und der Vollwertwerternährung ist in kontrollierten Studien weiter zu evaluieren.

Schlüsselwörter: Ernährungstherapie, Fasten, kalorische Restriktion, Mediterrane Ernährung, Naturheilkunde, Vollwerternährung

\section{Nutritional and Fasting Therapy in Complementary Medicine}

Background: There is growing evidence from epidemiological and clinical studies supporting the important role of nutrition and diet in the prevention and treatment of chronic disease. Moreover recent experimental data suggest that caloric restriction and intermittent fasting extend lifespan and prevent disease in rodents. Integrated therapeutic concepts that combine fasting therapy with health promoting nutrition are a cornerstone of traditional European naturopathy and complementary medicine. Objective: To examine from the literature to what extent nutritional therapies implementing concepts of traditional naturopathic medicine and intensified dietary interventions as fasting therapy and calorie restriction can contribute to the prevention and treatment of disease. Methods: The present selective review summarizes and rates the rapidly accumulating evidence from human studies (randomized trials, epidemiological and descriptive studies) and experimental investigations, coupled with information from nutritional standard works. Results and Conclusion: Traditional diets, such as the traditional Mediterranean diet, are highly effective in the treatment of coronary artery disease, the metabolic syndrome, type-2-diabetes mellitus and rheumatoid arthritis. It features an ovo-lacto vegetarian diet with a preference of fresh unheated foods processed as little as possible and demonstrates a variety of health-promoting effects. The combination of Mediterranean-type diet and wholesome nutrition shows good patient adherence and promising clinical effectiveness. Modified fasting therapy is a safe dietary intervention and efficacious in the treatment of rheumatoid arthritis and hypertension. Moreover, it enhances lifestyle modification and behavioral change of nutritional habits. Observational data point to potential effectiveness in the treatment of chronic pain syndromes, inflammatory and cardiovascular disease. Experimental data show distinct effects of intermittent fasting on neurodegenerative and age-associated disease. The clinical impact of fasting therapy and wholesome Mediterranean nutrition should be further evaluated in clinical trials.

Key words: Caloric restriction, Complementary medicine, fasting therapy, Mediterranean Diet, nutritional therapy, wholesome nutrition 
Tab. 1. Grundsätze der Vollwert-Ernährung und addititve Komponenten der mediterranen Kost

\section{Grundsätze der Vollwert-Ernährung}

1. Bevorzugung pflanzlicher naturbelassener Lebensmittel (überwiegend lacto-vegetabile Kost): Gemüse, Obst sowie Getreide, Hülsenfrüchte, Nüsse, Samen und Kräuter,

2. Vermeidung unnötiger Lebensmittelverarbeitung (Lebensmittel so natürlich wie möglich)

3. Etwa die Hälfte der Lebensmittel als unerhitzte Frischkost

4. Vermeidung von Lebensmittelzusatzstoffen und gentechnisch veränderten oder bestrahlten Lebensmitteln,

5. Nur selten und mässiger Verzehr von Fleisch, Eiern und Produkten mit hohem Anteil an gesättigten Fetten. Mässiger Verzehr von Fisch und Geflügel.

6. Weitgehender Verzicht auf Süsswaren, leicht verdauliche Kohlenhydrate und Convenience Food,

7. Verwendung von Lebensmitteln möglichst aus ökologischem Anbau sowie nach saisonalen und regionalen Gesichtspunkten.

8. Vermeidung aufwändiger Lebensmittelverpackung, Verwendung umweltverträglicher Produkte und Bevorzugung von Nahrungsmitteln aus sozialverträglicher Produktion

Additive Komponenten der mediterranen Kost

1. Ausschliessliche Verwendung von Olivenöl, Leinöl, Walnussöl, Rapsöl als Speiseöle

2. Reichliche Verwendung frischer Kräuter und Gewürze

3. Reichlicher Verzehr von Hülsenfrüchten, Linsen sowie von Nüssen und Mandeln

4. Verzehr von fettem Seefisch (Makrele, Sardine, Lachs, Hering) 1-2/x pro Woche soweit umweltverträglich

5. 5-7/ täglich Portionen von frischem Obst oder schonend zubereitetem Gemüse

naturheilkundlicher Ernährung beinhalteten im wesentlichen eine weitgehend vegetarische Kostform mit reichlichem Verzehr von Obst-, Gemüseund Getreide-Frischkost und Verzicht auf stark verarbeitete Lebensmittel.

Eine zeitgemässe Anpassung des Prinzips der Vollwerternährung unter Berücksichtigung ernährungsökologischer Gesichtspunkte erfolgte später durch die Giessener Arbeitsgruppe um LEITZMANN [5]. Hierbei wurden auch die ethischen, ökologischen und wirtschaftlichen Dimensionen von Ernährung angemessen berücksichtigt. In der Giessener Vollwertstudie konnten die positiven Wirkungen dieser Ernährungsform auf relevante Risikomarker und Surrogatparameter sowie die Praktikabilität der Umsetzung im Alltag belegt werden [6].

Die ernährungstherapeutischen Grundsätze der modernen naturheilkundlichen Vollwerternährung sind in Tabelle 1 zusammengefasst. Parallel zur ernährungsmedizinisch konzipierten Definition einer Vollwerternährung wurden von der Naturheilkunde auch Methoden zur praktischen Umsetzung und Sicherung der Patientenadhärenz entwickelt. Im Vordergrund stehen hier vor allem ordnungstherapeutische und gesundheitspädagogische Aspekte, die ihren Schwerpunkt weniger auf die Informationsvermittlung, sondern auf die praktische Erfahrung, das Wiedererlernen einer bewussten und achtsamen Nahrungsaufnahme und eine Steigerung der Selbstwirksamkeit im Prozess der Ernährungsumstellung setzen. Diese ordnungstherapeutischen Konzepte sind sowohl in ambulanten als auch stationären Behandlungskonzepten umsetzbar.

Von der naturheilkundlichen Vollwert-Ernährung abzugrenzen ist die sog. vollwertige Ernährung, wie sie von der Deutschen Gesellschaft für Ernährung (DGE) formuliert wird. Diese wird primär über eine bedarfsgerechte $\mathrm{Zu}$ sammensetzung der Nährstoffzufuhr definiert und berücksichtigt weder erweiterte medizinische noch ökologische Gesichtspunkte von Ernährung. Hierbei gilt eine Kost dann als vollwertig, wenn sie alle nötigen Nährstoffe in ausreichender Menge, im richtigen Verhältnis und in der richtigen Form enthält. Die vollwertige Ernährung der DGE ist nicht primär vegetarisch.

In der klinischen Naturheilkunde wurde der Ernährung konstant eine vorrangige Bedeutung für die Therapie und Prävention chronischer Erkrankungen zuerkannt. Demgegenüber führten Ernährungsmedizin und Ernährungstherapie in der konventionellen klinischen Medizin bis in die 80er Jahre des letzten Jahrhunderts ein Schattendasein. Die Ergebnisse der grossen internationalen epidemiologischen Studien (Nurses Health Study, Framingham Study, Physicians Health Study u.a.) und die daraus abgeleiteten Risikofaktormodelle für kardiovaskuläre und onkologische Erkrankungen belegten aber zunehmend die überragende Bedeutung gesunder Ernährung für die Gesunderhaltung und bestätigten damit auch zahlreiche theoretische Grundannahmen zur Vollwerternährung. Während die konventionelle Ernährungsmedizin sich zuvor vorwiegend mit den Problemen der Mangel- und Unterernährung beschäftigte, wurde nun deutlich, dass etwa zwei Drittel aller massgeblichen Erkrankungen der modernen Industriegesellschaft direkt oder indirekt ernährungs- und lebensstilabhängig sind. Die herausragende Bedeutung der Ernährung als pathogenetischer Faktor ist inzwischen für eine Vielzahl der massgeblichen Zivilisationskrankheiten eindeutig belegt, u.a. für die koronare Herzerkrankung, Diabetes mellitus, arterielle Hypertonie, Schlaganfall, Mammakarzinom, Prostatakarzinom und das Kolonkarzinom. Insbesondere aber wurde auch erkennbar, dass für die Epidemie der Fehlund Überernährung keine praktikablen und wirksamen therapeutischen Konzepte in der konventionellen Medizin vorliegen.

Aktuell publizierte Daten aus epidemiologischen Studien legten schliesslich nahe, dass die Bedeutung der Ernährung für die Entstehung und Progression von Herz-Kreislauf Erkrankungen sogar noch unterschätzt wurde. So belegt die INTERHEART Studie, dass etwa 90\% aller Herzinfarkte durch Faktoren des Lebensstils, und hier vorrangig der Ernährung, erklärt werden können [7]. Vermutlich wurde auch in anderen Indikationsbereichen der Einfluss von Ernährung eher unterschätzt, da bekannte methodische Limitierungen von Ernährungs- 
studien wie „underreporting“, falsche Einstufung von Nahrung in Kantinen und Restaurants oder die mangelnde Differenzierung von Nahrungsmitteln (z. B. Fettsäuren) zur Verdünnung von Effekten führt.

Die aktuell massive Zunahme der Adipositas bereits im Kinder- und Jugendalter unterstreicht die zukünftige Bedeutung erweiterter ernährungsmedizinischer Ansätze. Der Begriff „toxic environment“ beschreibt die Verbindung von „poor nutrition“ und „low physical activity“ und kritisiert die massive Marketingaktivität der Ernährungsindustrie, die besonders auf Kinder abzielt. Aktuelle neurobiologische und neuroendokrine Forschungen konnten zeigen, dass das konventionelle Nahrungsangebot der Ernährungsindustrie durch ungünstige Wirkungen auf Insulinspiegel, Leptin und die zentrale Appetitregulation komplexe negative Wirkungen auf Ernährungsverhalten und den Lebensstil induziert [8]. Dies impliziert, dass die ernährungsmedizinischen Fortschritte die epidemiologische Entwicklung hier nicht ausreichend beeinflussen können, sondern gesellschaftliche und politische Einflussnahme notwendig ist.

\section{Die Mediterrane Ernährung (Mittelmeerkost)}

Bereits in frühen Daten der SiebenLänder-Studie fiel auf, dass Länder mit traditionellen Ernährungsformen wie Japan und einzelne Mittelmeerregionen (Kreta, Sizilien) eine signifikant erniedrigte Inzidenz an Herz-Kreislauf-Erkrankungen und Krebserkrankungen aufwiesen [9]. Inzwischen wurden für viele typische Nahrungsmittel der sog. traditionellen Mittelmeerkost gesundheitsfördernde Effekte nachgewiesen. Durch den hohen Anteil an pflanzlicher Frischkost, Obst, Hülsenfrüchten, Wurzelgemüse, Nüssen und Samen sowie dem reduzierten Verzehr von Fleisch und Wurst finden sich sowohl in den traditionellen asiatischen als auch mediterranen Ernährungsformen zahlreiche inhaltliche Übereinstimmungen mit dem Konzept der Vollwerternährung der Naturheilkunde.
Darüber hinaus erscheint aber ein wesentlicher Anteil der gesundheitsfördernden Wirkungen der Mittelmeerkost speziell durch die dort verwendeten Öle und Fette vermittelt. Bereits in der Sieben-Länder-Studie fiel auf, dass die mediterrane Kost nicht als fettarm, sondern eher fettreich anzusehen ist (Nahrungsenergieanteil von $40 \%$ durch Fett), was damals auch den Studienleiter KEYs zur Anmerkung veranlasste, dass dort (in Kreta) Olivenöl eher als Getränk, denn als Speise anzusehen sei [9]. In der traditionellen Mittelmeerkost werden als Fettsäuren überwiegend langkettige Omega-3 Fettsäuren aus Fisch (EPA, DHA) sowie kurzkettige pflanzliche Omega-3 Fettsäuren aus Walnüssen, Leinsamen und spezifischen Gemüsesorten sowie einfach gesättigte Omega-9 Fettsäuren aus Olivenöl, Nüssen, Avocados und Mandeln zugeführt. Omega 6-Fettsäuren (v.a. in konventionellen Speiseölen) und gesättigte Fette aus Fleisch, Wurst, Milch und Milchprodukten werden hingegen in nur geringem Masse konsumiert. Hierbei wird ein günstiges Verhältnis von Omega-6/Omega-3 Fettsäuren von 5:1 erreicht, während dies bei konventioneller westlicher Ernährung bei 10:1 bis 20:1 liegt.

Die Fettsäurezusammensetzung von Milchproduktem muss hierbei nicht zwingend ungünstig sein. Eine Schweizer Arbeitsgruppe konnte beispielsweise unlängst nachweisen, dass Bergkäse, der durch traditionelle Sennerwirtschaft hergestellt wird, eine stark abweichende Fettzusammensetzung mit deutlich höheren Anteilen an protektiven Omega-3 Fettsäuren, im Vergleich zu herkömmlichem Flachlandkäse, aufweist [10]. Bekannt ist auch, dass die Fettsäurezusammensetzung von Fleisch und Wurst wesentlich von den Viehfütterungs- und Haltungsmethoden abhängt [11]. Dies unterstreicht ernährungsökologische Sichtweisen, wonach sich natürliche Herstellungsbedingungen auch positiv auf die Nährstoffzusammensetzung von Lebensmitteln auswirken.

Umfangreiche Studiendaten belegen inzwischen spezifische medizinische Wirkungen einzelner charakteristischer Nahrungsmittel der Mittelmeerdiät. Beispielhaft genannt seien die blut- drucksenkende Wirkung von Olivenöl [12], die Verbesserung der Gefässendothelfunktion durch Walnüsse [13], die Stabilisierung arteriosklerotischer Placques durch Fischöl [14] sowie die Verbesserung des Fettstoffwechsels durch Mandeln [15]. Die nachgewiesene protektive Wirkung der Fischöle DHA und EPA in der Primär- und Sekundärprävention des Herzinfarktes, des plötzlichen Herztodes und des Bluthochdrucks hat inzwischen zu Empfehlungen zur Supplementierung von Fischöl in der Sekundärprävention nach Herzinfarkt durch die American Heart Association geführt [16]. Auch moderater Weinkonsum zu den Mahlzeiten ist Bestandteil der traditionellen mediterranen Ernährung, Die gesundheitsfördernden Wirkungen insbesondere von Rotwein sind inzwischen umfangreich belegt [17]. Von Bedeutung erscheint, dass Polyphenole des Rotweins (Resveratrol) spezifisch sog. Sirtuine (silent information regulator) aktivieren. Sirtuine sind NAD+-abhängige Deacetylasen und regulieren experimentell den zellulären Fettstoffwechsel, inflammatorische zelluläre Reaktionen, schützen vor neuronalem Zelltod und regulieren die Lebensspanne von Organismen [18,19]. Welche Bedeutung dem Weinkonsum allerdings in der protektiven Gesamtwirkung der mediterranen Ernährung zukommt, bleibt derzeit unklar.

Auch erlauben bislang epidemiologischen Daten keinen Rückschluss, ob sich weitere assoziierte Faktoren des mediterranen Lebensstils zu den ernährungsphysiologischen Effekten addieren. Zuletzt konnte beispielsweise eine kardiovaskuläre Risikoreduktion durch die Praxis der „Siesta“ aufgezeigt werden [20]. Auch auf die mögliche medizinische Bedeutung der Esskultur und der zeitlichen Bedingungen der Nahrungsaufnahme wurde zuletzt hingewiesen. In diesem Zusammenhang wurde die Abhängigkeit der Verdauung und damit der Nährstoffaufnahme von neuropsychologischen Faktoren mit dem Begriff „Vitamin T“ (time) belegt [21]; dieser Ansatz reflektiert auch aktuelle Strömungen in Gastronomie und Landwirtschaft wie „slow food“ und entspricht ordnungstherapeutischen Empfehlungen zur Nahrungsaufnahme. 
Die Wirksamkeit der mediterranen Ernährung als Gesamternährungsform wurde inzwischen in mehreren randomisierten Studien für verschiedene Indikationen beeindruckend nachgewiesen. Im Lyon Diet Heart Trial wurden Herzinfarktpatienten einer mediterranen Diät oder einer konventionellen Diät randomisiert zugeordnet. Die Studie musste vorzeitig abgebrochen werden. Es zeigte sich eine relative Risikoreduktion für kardiovaskuläre Ereignisse und die Gesamtmortalität von etwa $70 \%$ [22,23], aber auch eine verringerte Inzidenz an Krebserkrankungen. In der Lyon Diet Heart Studie erfolgte die Zufuhr von pflanzlichen Omega-3 Fettsäuren auch über eine reine Rapsölmargarine. Die Verwendung von Rapsöl kann somit auch im Rahmen der mediterranen Kost Eingang finden, im Konzept der traditionellen mediterranen Kost ist allerdings Rapsöl für die ausreichende Omega-3 Fettsäurenzufuhr nicht erforderlich. In der INDO-Mediterranean Heart Study wurden Nahrungskonzepte der mediterranen und asiatischen Kost kombiniert. Auch bei dieser randomisierten Studie mit 1000 Patienten zeigte sich eine signifikante Absenkung an kardiovaskulären Folgeereignissen im Studienzeitraum von 2 Jahren [24]. Inzwischen wurden die günstigen Wirkungen einer mediterranen Kost auch für die Therapie der rheumatoiden Arthritis [25] und des Diabetes mellitus [26] in randomisierten Studien belegt. Eine weitere Studie untersuchte die Wirksamkeit der mediterranen Kost bei metabolischem Syndrom. Hier zeigte sich nach 2 Jahren mediterraner Kost eine erfolgreiche Therapie des metabolischen Syndroms bei über $50 \%$ der so ernährten Patienten und parallel eine signifikante Reduktion relevanter inflammatorischer Risikomarker (HS, CRP, Interleukin 18) [27]. Darüber hinaus weisen Daten der HALE-Studie auf einen möglichen lebensverlängernden Effekt der mediterranen Diät [28] hin. Für die Anwendung in der Therapie der Hypercholesterinämie erscheint von Bedeutung, dass eine mediterrane Diät die Wirkung der medikamentösen Therapie mit Statinen potenziert und gleichzeitig mögliche negative Effekte der Sta- tine auf die antioxidative Kapazität $\mathrm{zu}$ kompensieren vermag [29].

\section{Die Mediterrane Vollwerternährung}

Inzwischen zeigten kontrollierte ernährungsmedizinische Studien auch für einzelne Elemente der naturheilkundlichen Vollwerternährung gesundheitsfördernde Wirkungen auf. So fanden sich bei Verzehr von Vollkorngetreideprodukten gegenüber verarbeitetem Getreide eine verbesserte Insulinsensitivität [30], ein reduziertes Risiko für kardiovaskuläre Ereignisse [31], ein verbessertes metabolisches Profil [32], reduzierte Blutdruckwerte [33] sowie potentielle antikanzerogene Wirkungen [34,35].

Erhöhte Anteile von Frischkost erwiesen sich wirksam in der Behandlung der rheumatoiden Arthritis [36, 37] und des Bluthochdrucks [38]. Weiter konnte aufgezeigt werden, dass durch zu starkes und langes Erhitzen von Eiweissen in Kombination mit Fetten und Zucker für den Körper nicht mehr verstoffwechselbare sog. Glykierungsendprodukte (Advanced glycation endproducts, AGEs) entstehen, die wiederum deutlich mit dem Auftreten und Fortschreiten von arteriosklerotischen Folgeerkrankungen, Diabetes mellitus und Nierenerkrankungen assoziiert sind [39]. Eine wenig erhitzte (AGE-arme) Ernährung zeigte in ersten Interventionsstudien im Vergleich zu identischer, aber stark erhitzter Kost (AGE-reich) signifikant günstige Effekte auf die Funktion von Adipozyten und das Gefässendothel [40,41].

Rückschlüsse auf günstige medizinische Wirkungen vollwertiger Kostformen können auch aus vorliegenden Daten zu verwandten und methodisch gut evaluierten Ernährungsformen gezogen werden. So beinhaltet die von ORNISH entwickelte fettarme vegetarische Kostform einen ausgeprägt hohen Frischkostanteil in Form von Gemüse und Obst. Die Wirksamkeit der Ornish Diät in der Behandlung der koronaren Herzerkrankung ist umfangreich belegt $[42,43]$, in einer Pilotstudie zeigten sich zudem günstige Wirkungen bei fortgeschrittenem Prostatakarzinom [44]. In der Behandlung des Bluthochdrucks hat sich im amerikanischen Raum die DASH Diät etabliert [45,46], wiederum eine Kostform mit hohen Frischkostanteilen von Obst und Gemüse und Verwendung fettreduzierter Milchprodukte. Schliesslich konnte eine Regression von arteriosklerotischen Veränderungen durch eine multimodale ayurvedische Intervention mit Schwerpunkt auf eine frischkostreiche vegetarische Ernährung belegt werden [47].

Vor diesem Hintergrund wurde an der eigenen Klinik das Konzept der sog. Mediterranen Vollwerternährung entwickelt. Hierbei wird die Vollwerternährung nach Leitzmann insbesondere durch die ausschliessliche Verwendung von Olivenöl, Leinöl und Rapsöl als Speiseöle, aber auch durch den Verzehr von Nüssen und Mandeln sowie ein- bis zweimal pro Woche Fisch und die reichliche Verwendung von aromatischen Kräutern und Gewürzen ergänzt [48]. In der klinischen Ernährungsberatung zeigt diese Ernährungsform durch ihre hohe Praktikabilität und Schmackhaftigkeit eine hohe Akzeptanz und Umsetzbarkeit. Langzeitdaten aus einer eigenen Beobachtungsstudie zeigen auch 12 Monate nach Entlassung aus dem Krankenhaus eine gute Ernährungsadhärenz und eine gesteigerte Selbstwirksamkeit in Bezug auf das Ernährungsverhalten bei der Mehrzahl der behandelten Patienten [49]. In einer eigenen randomisierten Studie an Koronarpatienten bestätigte sich die gute Adhärenz. Innerhalb eines multifaktoriellen Behandlungsprogramms führte die Mediterrane Vollwerternährung zu Verbesserungen der Lebensqualität, der kardialen autonomen Funktion, der Blutdruckregulation und zur Reduktion von Angina pectoris Beschwerden [50].

Insbesondere erscheint die traditionelle mediterrane Kost auch als eine geeignete Ernährungsform für die Prävention und Therapie des metabolischen Syndroms. Die Ineffektivität und mangelnde Nachhaltigkeit von Diätprogrammen zur Gewichtsabnahme wurde zuletzt deutlich aufgezeigt. In einer randomisierten Studie 
erwiesen sich vier bekannte Diätprogramme zur Gewichtsabnahme (u.a. weight watchers, Atkins) bereits nach mehreren Monaten als völlig ineffektiv mit Compliance-Raten von etwa $30 \%$ nach einem Jahr unter allen Diätformen [51]. Im Gegensatz hierzu erscheint die Kombination einer vollwertigen mediterranen Ernährung mit ordnungstherapeutischen Massnahmen in der Therapie der Adipositas und des metabolischen Syndroms äusserst vielversprechend. Allerdings ist auch hier die weitere Evaluation in kontrollierten Studien notwendig.

\section{Therapeutisches Fasten („Heilfasten“)}

Fasten, definiert als der freiwillige und zeitlich definierte Verzicht auf feste Nahrung und Genussmittel, hat weltweit eine umfassende ethnokulturelle und religiöse Tradition. In diesem Kontext wird die intensive Erfahrung des Nahrungsverzichts vor allem zur Vertiefung der religiösen und spirituellen Praxis eingesetzt. Noch heute sind Fastentage und Fastenzeiten in vielen Religionen etabliert (z.B. Yom Kippur, Ramadan, Christliche Fastenzeit), wenngleich zumeist die Fasteninhalte gelockert wurden und zumeist nur noch partiell der ursprünglichen Fastentradition entsprechen.

Jahreszeitlich bedingte Schwankungen im Nahrungsangebot prägten in der Evolution den Stoffwechsel aller Lebewesen. Die Fähigkeit, sich diesen Schwankungen durch Depotbildungen und deren Mobilisierung anzupassen, war von jeher Bedingung für das menschliche Überleben. Sie bildet die physiologische Grundlage für die Fähigkeit, für eine begrenzte Zeit den Bedarf an Mikro- und Makronährstoffen bei ausbleibender oder nur minimaler Nahrungsaufnahme ohne gesundheitliche Nachteile aus körpereigenen Reserven zu decken. Darüber hinaus kann aufgrund einer Vielzahl von inzwischen vorliegenden wissenschaftlichen Daten hypothetisiert werden, dass periodische Fastenzeiten zusätzliche therapeutische bzw. gesundheitsfördernde Effekte haben. Letztere umfassen sowohl metabolische als auch neuroendokrine und psychologische Effekte. Aus der biologischen Fähigkeit entwickelten sich weltweit Fastenkulturen mit religiös-spiritueller als auch medizinisch-therapeutischer Zielsetzung. In der Entwicklung ab dem 20. Jh. integrierte sich die Fastentherapie bzw. das therapeutische Fasten oder „Heilfasten“ über die Naturheilkunde in die Medizin. Insbesondere im deutschsprachigen europäischen Raum, in Skandinavien und in der Indischen Medizin stellt das Fasten heute eine etablierte Methode in Therapie und Prävention dar.

Als Fastenformen haben sich im deutschsprachigen Raum vor allem die modifizierten Methoden nach Dr. Otto Buchinger (Erstveröffentlichung 1935) und nach Dr. F.X. Mayr (1921) durchgesetzt. Fastenvarianten sind das Molkefasten und das Schleimfasten. Vom modifizierten Fasten, das sich durch eine geringe, aber spezifizierte Nährstoffzufuhr unterhalb einer Nahrungs-Energiezufuhr von $500 \mathrm{kcal} / \mathrm{Tag}$ und ein komplexes therapeutisches Setting auszeichnet, sind die physiologisch nachteiligen Nulldiäten sowie die grosse Menge der Crash-Diäten abzugrenzen.

Daten aus Grundlagenstudien und wissenschaftlichen Qualitätssicherungen liegen inzwischen vor allem für die Methode nach Buchinger vor. Buchinger entwickelte aus rudimentären Ansätzen ein umfassendes multidisziplinäres Konzept für eine stationäre Fastentherapie, in der Physio-, Bewegungs-, und dem Fasten nachfolgende Ernährungstherapie mit einem ordnungstherapeutischen Programm kombiniert sind [52,53,54]. Die Inhalte des therapeutischen Fastens wurden 2002 in einer Leitlinien-Konsensus-Konferenz unter Leitung der Ärztegesellschaft für Heilfasten und Ernährung (ÄGHE; www.aeghe.de) zusammengefasst und publiziert [55].

\section{Indikation und \\ Wirksamkeitsnachweis des therapeutischen Fastens}

Die wesentlichen Kennzeichen des Buchinger-Fastens sind in Tabelle $2 \mathrm{zu}-$ sammengefasst. Als wesentliche Indi- kationen des therapeutischen Fastens haben sich insbesondere die Krankheitsbilder der rheumatoiden Arthritis und der chronifizierten Schmerzsyndrome einschliesslich Migräne/Fibromyalgie herausgebildet. Weitere Hauptindikationen sind: Metabolisches Syndrom und Übergewicht, stabile Formen der Herz- und Gefäss-Erkrankungen, Asthma und Allergien, Hauterkrankungen und Magen-Darm-Erkrankungen [56]. Zu betonen ist, dass das primäre Ziel des therapeutischen Fastens nicht die daraus resultierende Gewichtsabnahme, sondern die Induktion spezifischer und nachhaltiger therapeutischer Effekte ist. Bei ausgeprägter Adipositas (BMI $>45 \mathrm{~kg} / \mathrm{m}^{2}$ ) ist durch die temporäre fasteninduzierte Absenkung des Grundumsatzes ein Jojo-Effekt potentiell induzierbar. Allerdings belegen Studiendaten, dass auch bei ausgeprägter Adipositas ein günstiger Langzeiteffekt mit stationärem Fasten $\mathrm{zu}$ erzielen ist, also bei sachgerecht durchgeführter Fastentherapie kein JoJo Effekt eintritt [57,58,59] Als relative Kontraindikation des Fastens sind hingegen Formen von Adipositas per magna mit zugrundeliegender Essstörung (Binge eating disorder) aufzufassen.

Insbesondere für den Indikationsbereich der rheumatischen Erkrankungen konnten mehrere kontrollierte Studien die Wirksamkeit des Fastens eindeutig belegen. So konnten KJELDSEN-KrAGH et al. von der Universität Oslo in einer im Lancet publizierten randomisiert-kontrollierten Studie zeigen, dass bei Patienten mit rheumatoider Arthritis eine einwöchige Fastentherapie, gefolgt von einem Kostaufbau zu veganer und nachfolgend lactovegetarischer Ernährung zu einer ausgeprägten Beschwerdebesserung und zum Rückgang von Entzündungsparametern über etwa ein Jahr führt [60]. Die klinische Wirksamkeit der Fastentherapie bei rheumatoider Arthritis wurde inzwischen durch mehrere kontrollierte Studien und in einer systematischen Übersicht bestätigt [61]. Als mögliche Mechanismen der antirheumatischen Wirkung werden u.a. eine fasteninduzierte Leptindepletion mit konsekutiver T2 Lymphozytensuppression [62], die endogene Hypercortisol- 
Tab. 2. Fastenmethode

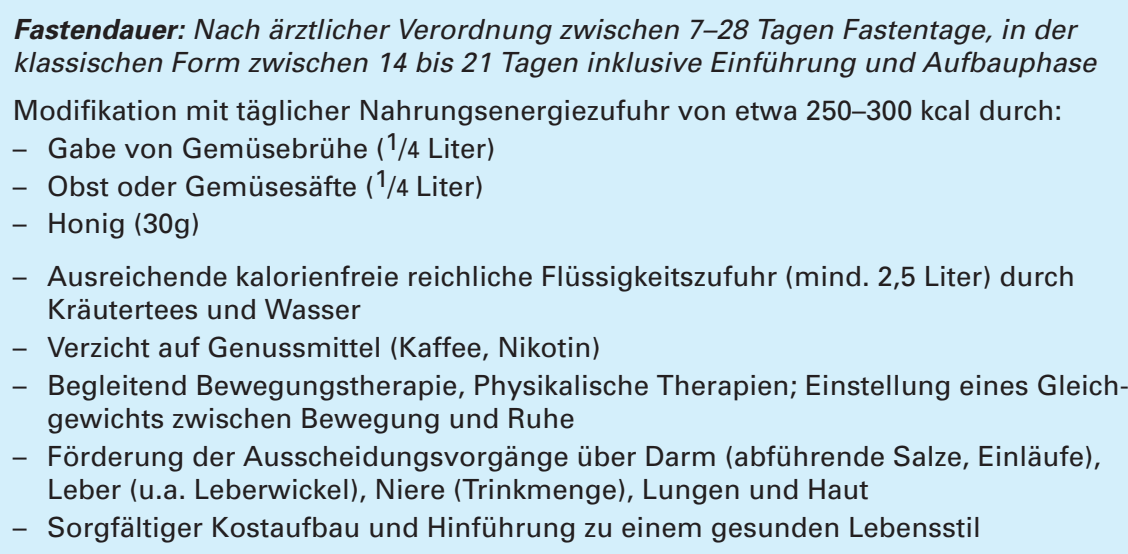

Fastendauer: Nach ärztlicher Verordnung zwischen 7-28 Tagen Fastentage, in der klassischen Form zwischen 14 bis 21 Tagen inklusive Einführung und Aufbauphase

Modifikation mit täglicher Nahrungsenergiezufuhr von etwa 250-300 kcal durch:

- Gabe von Gemüsebrühe ( $1 / 4$ Liter)

- Obst oder Gemüsesäfte ( $1 / 4$ Liter)

- Honig (30g)

- Ausreichende kalorienfreie reichliche Flüssigkeitszufuhr (mind. 2,5 Liter) durch Kräutertees und Wasser

- Verzicht auf Genussmittel (Kaffee, Nikotin)

- Begleitend Bewegungstherapie, Physikalische Therapien; Einstellung eines Gleichgewichts zwischen Bewegung und Ruhe

- Förderung der Ausscheidungsvorgänge über Darm (abführende Salze, Einläufe), Leber (u.a. Leberwickel), Niere (Trinkmenge), Lungen und Haut

- Sorgfältiger Kostaufbau und Hinführung zu einem gesunden Lebensstil

\section{Variationen:}

Zugabe von Buttermilch für längere Fastendauern

Molkefasten (täglich 0,5 Liter Kurmolke)

Schleimfasten (Hafer oder Buchweizen; insbesondere bei Magenempfindlichkeit) ämie als Folge der metabolischen Anpassungsreaktion [63] sowie komplexe Effekte auf das intestinale Immunsystem (GALT) diskutiert [64,65].

Daten aus grösseren Beobachtungsstudien [58,66] und konsistente Erfahrungen in fast allen spezialisierten Fastenkliniken weisen auch auf einen relevanten therapeutischen Effekt des Fastens bei chronischen Schmerzerkrankungen des Bewegungsapparates hin. Teilweise scheint dies auch durch die nach einer Fastentherapie verbesserte Umsetzung einer gesundheitsfördernden Lebensstilmodifikation (Bewegung, Entspannungsverfahren, nachfolgende Ernährungsumstellung) vermittelt [66].

Tierexperimentell sind aber auch eine zentrale Hypoalgesie und direkte antinocizeptive Effekte infolge kalorischer Restriktion oder intermittierendem Fasten belegt [67]. In einer unkontrollierten Therapiestudie wurde auch eine gute Wirksamkeit des Fastens bei Migräne beschrieben [68]. Experimentell wurde nach ein- und zweiwöchigem Fasten bei Versuchstieren entsprechend eine erhöhte zentrale Serotoninverfügbarkeit nachgewiesen [69], was sowohl ein mögliches Erklärungsmodell für die therapeutische Wirkung bei Migräne ergibt, als auch die klinisch belegten stimmungsaufhellenden Wirkungen des Fastens [70] und die belegte Modulation der Schlaf- architektur [71] erklären könnte. Schliesslich induzieren Fastenperioden ausgeprägte Modulationen der nebennierenrindenabhängigen Katecholaminproduktion [72,73,74]. Initial kommt es unter Fasten zu einem deutlichen Anstieg der Adrenalin-, Noradrenalin- und Cortisol-Spiegel [75 , im weiteren Verlauf finden sich hingegen reduzierte Katecholaminspiegel, auch unter körperlicher Stressinduktion [76]. Ein schmerzreduzierender Effekt dieser sog. „vegetativen Umstimmung“ ist naheliegend, da das autonome Nervensystem massgeblich bei der Schmerzempfindung beteiligt ist.

Ausführlich belegt sind die antihypertensiven Effekte des Fastens [75, $77,78,79]$. Trotz der initialen endokrinen Stressreaktion resultiert durch die ausgeprägte Natriurese des Fastens und die Ausschüttung von natriuretischem Peptid [80] eine rasche und ausgeprägte fasteninduzierte Blutdrucksenkung. Klinisch ist hierbei auf eine Dosisreduktion der antihypertensiven Medikation zu achten. Nach dem Fasten kommt es zumeist $\mathrm{zu}$ einem leichten Wiederanstieg der Blutdruckwerte, die jedoch nicht die Höhe der Ausgangswerte erreichen, auch fortgeführt finden sich zumeist über mehrere Monate verbesserte Blutdruckwerte. Verantwortlich für diesen nachhaltigen Effekt erscheint, neben der resultierenden Gewichtsabnahme und nachfolgenden Ernährungsumstellung, eine Sensibilisierung von Rezeptoren der Blutdruckregulation, insbesondere des atrialen natriuretischen Peptids [77].

Zur Wirksamkeit der Fastentherapie bei koronarer Herzkrankheit liegen keine randomisierten Studien vor, jedoch lassen die aufgezeigten günstigen metabolischen, endokrinen und antihypertensiven Effekte und inzwischen vorliegende tierexperimentelle Daten [81] auf einen potentiellen Nutzen schliessen.

\section{Anti-Aging-Effekte des Fastens?}

Tierexperimentell ist seit längerem bekannt, dass eine nutritive Kalorienrestriktion von etwa $30 \% \mathrm{zu}$ einer Lebensverlängerung verschiedener Spezies führt [82]. „Kontrollierte“ Unterernährung (ohne Fehlernährung) stellt damit die bislang einzige bekannte Methode dar, die tatsächlich „AntiAging“-Effekte aufweist und mit einer Lebensverlängerung verbunden ist [83]. Neben einer Kalorienrestriktion einzelner Mahlzeiten wurden experimentell solche Wirkungen auch durch Reduktion der Anzahl täglicher Mahlzeiten (z.B. Dinner cancelling) oder durch intermittierendes Fasten erzeugt. Beispielsweise zeigen Mäuse und Ratten, die ein intermittierendes Fastenschema erhalten (24 Stunden Essen ad libitum und 24 Stunden Fasten im Wechsel) eine 30\%ige längere Lebenserwartung als kontinuierlich täglich gefütterte Versuchstiere [84]. Kalorienrestriktion und intermittierendes Fasten führen so (unabhängig vom resultierenden Gewicht) $\mathrm{zu}$ Verbesserungen des Glukosestoffwechsels, der Insulinsensitivität, senken den Blutdruck, verbessern die kardiovaskuläre Stressresistenz, die altersreduzierte Nierenfunktion sowie die Immunfunktion und Anfälligkeit für neurodegenerative Gehirnerkrankungen wie Morbus Parkinson oder Alzheimer Demenz [84].

Erstaunlicherweise gibt es bislang jedoch kaum klinische Studien am Menschen, die den Einfluss der Mahlzeitenfrequenz in Prävention und The- 
rapie untersucht haben [85]. In wenigen ersten Untersuchungen zum (intermittierenden) Ramadan-Fasten konnten einzelne Verbesserungen von Risikomarkern am Ende des Ramadans dokumentiert werden, beispielsweise erhöhte HDL-Cholesterinwerte und verringerte LDL-Cholesterinwerte und Blutgerinnungsneigung [85].

Eine unlängst in JAMA publizierte kontrollierte Studie untersuchte erstmals den Effekt kontinuierlicher kalorischer Restriktion oder initialer very low calorie diet (VLCD, tägliche Energiezufuhr <900 kcal mit definierten, nicht naturbelassenen Nährstoffgemischen) auf Marker der biologischen Alterung bei leicht übergewichtigen Probanden. Es zeigte sich sowohl unter kalorischer Restriktion als auch unter VLCD, gefolgt von normokalorischer Kost, nach 6 Monaten eine verbesserte Insulinsensitivität und bemerkenswerterweise eine Reduktion der altersprogredienten DNA- Schädigung [86]. In weiteren Studien ist $\mathrm{zu}$ überprüfen, ob solche Effekte unter Fastentherapie möglicherweise noch ausgeprägter sind.

Verschiedenste mögliche Mechanismen werden für die „Anti-Aging“-Wirkung des experimentellen intermittierenden Fastens diskutiert, so die Induktion neurotropher Faktoren [87], die vermehrte Ausschüttung von Wachstumshormon [88,89] und die Aktivierung von sog. Sirtuinen (s. oben) [19,90) und der verminderte Anfall von radikalen Sauerstoffspezies.

Unklar ist derzeit, ob Fasten auch eine Einfluss auf die Belastung durch AGEs (s.o.) hat. SELL und Mitarbeiter wiesen eine kurvi-lineare Beziehung zwischen mit dem Lebensalter ansteigenden AGE-Konzentrationen der Haut und der jeweiligen durchschnittlichen Lebenserwartung der Spezies nach [91]. Je niedriger die altersproportionale AGE-Konzentration im Hautgewebe war, desto länger war die Lebenserwartung. Darüber hinaus zeigte sich, dass Versuchstiere, die eine kalorische Restriktion oder ein intermittierendes Fasten erhielten, eine geringere AGE-Akkumulation und konsekutiv die bekannte Verlängerung der durchschnittlichen Lebensspanne zeigten [92]. Ob diese Zusammenhänge kausal sind und auf den Menschen übertragbar sind, muss allerdings zum jetzigen Zeitpunkt unklar bleiben.

Zum anderen zeigten zuletzt mehrere Studien, die die zelluläre Körperreaktion auf Nahrungszufuhr untersuchten, dass jede Nahrungszufuhr, unabhängig vom Gehalt an Fett, Eiweiss, Kohlenhydraten oder AGEs zu einer signifikanten zellulären Stressreaktion mit Aktivierung des Transkriptionsfaktors NF-KB führt [93,94] und ein Korrelat der bekannten postprandialen Leukozytose darstellt. Dies würde implizieren, dass jede Reduktion an eingenommenen Mahlzeiten für den Körper mit einer reduzierten zellulären Stressbelastung verbunden ist. Darüber hinaus scheinen Fastenperioden $\mathrm{zu}$ einer verbesserten zellulären Stress-Resistenz zu führen [85]. Schliesslich sind komplexe Wirkungen des Fastens auf das Immunsystem belegt und auch die anorektische Antwort bei akuten Erkrankungen ist mit günstigen Wirkungen auf die Immunkompetenz assoziiert [95].

Klinisch kann das Fasten als eine äusserst sichere Therapiemethode angesehen werden. Anekdotische und immer wieder in der Laienpresse zitierte einzelne Todesfälle infolge von Extrem-Perioden (>12 Wochen) von Nulldiäten sind in früher Literatur beschrieben und keinesfalls auf die Methode des therapeutischen Fastens übertragbar. Bei sachgerechter Durchführung des Fastens sind keine ernsthaften Nebenwirkungen oder gar Todesfälle bekannt. Leichteren Nebenwirkungen des Fastens wie initialer Kopfschmerz, vermehrte Kälteempfindlichkeit, Kreislaufbeschwerden durch niedrigen Blutdruck kann durch einfache Gegenmassnahmen begegnet werden.

Kritisiert wird das Fasten vielfach von ernährungsphysiologischer Seite aufgrund seiner proteinkatabolen Wirkungen und des möglichen konsekutiven Muskelabbaus. Durch Zugabe kleiner Mengen Kohlenhydrate oder Eiweiss (Obst-,Gemüsesäfte, Honig, gel. Milchprodukte) bei den bekannten Fastenmethoden wird dieser Eiweissabbau deutlich reduziert [96,97]. Darüber hinaus konnte gezeigt werden, dass der fasteninduzierte Anstieg der Wachstumshormonspiegel eine Inhibition des Muskeleiweiss-Abbaus bewirkt [98]. Ob der geringe resultierende Eiweiss-Abbau des Fastens medizinisch noch mit günstigen oder ungünstigen Wirkungen behaftet ist, ist ungeklärt, erscheint aber in Kenntnis der umfangreichen Daten aus Beobachtungsstudien [58,66,99,100] nicht von klinischer Relevanz.

\section{Fazit}

Zusammenfassend kann die Kombination aus einer traditionell mediterranen Diät und der in der Naturheilkunde entwickelten Vollwerternährung als eine empfehlenswerte Ernährungsform für die Prävention und Therapie der häufigsten Erkrankungen der Industriegesellschaften gewertet werden. Das therapeutische Fasten ist eine wirksame additive Therapie in der Behandlung der rheumatoiden Arthritis und chronischer Schmerzsyndrome und erscheint vielversprechend in der Behandlung metabolischer Erkrankungen, des Bluthochdrucks und der stabilen koronaren Herzerkrankung. Darüber hinaus stellt das Fasten eine geeignete Methode zur Einleitung einer Lebensstil- und Ernährungsumstellung dar. Das Potential dieser ernährungstherapeutischen Methoden, auch für weitere Erkrankungen, sollte umfassender genutzt und in kontrollierten Studien weiter evaluiert werden.

\section{Literatur}

1. Rotschuh KE. Naturheilbewegung, Reformbewegung, Alternativbewegung Stuttgart: Hippokrates; 1983.

2. Bircher-Benner M. Grundzüge der Ernährungstherapie Berlin; 1903.

3. Kollath U. Der Vollwert der Ernährung Heidelberg: Haug; 1987.

4. Anemueller H. Richtig essen München: DTV Ratgeber; 1994

5. Leitzmann C. Vollwerternährung, Ernährungsökologie und Lebensmittelqualität. In: Melchart D, Wagner H, eds. Naturheilverfahren. Stuttgart: Schattauer; 1993.

6. Hoffmann I, Groeneveld MJ, Boeing H, Leitzmann C. Giessen Wholesome Nutrition Study: relation between a health-conscious diet and blood lipids. Eur J Clin Nutr. 2001;55: 887-895.

7. Yusuf S, Hawken S, Ounpuu S, et al. Effect of potentially modifiable risk factors associated with myocardial infarction in 52 countries (the INTERHEART study): case-control study. Lancet. 2004;364(9438):937-52. 
8. Lustig RH. Childhood obesity: behavioral aberration or biochemical drive? Reinterpreting the First Law of Thermodynamics. Nat Clin Pract Endocrinol Metab. 2006;2(8):447-58.

9. Keys AB. Seven countries: a multivariate analysis of death and coronary heart disease. Cambridge, Mass.: Harvard University Press; 1980.

10. Hauswirth CB, Scheeder MR, Beer JH. High omega-3 fatty acid content in alpine cheese: the basis for an alpine paradox. Circulation. 2004; 109(1):103-7.

11. Crawford MA. Fatty-acid ratios in free-living and domestic animals. Lancet. 1968:1:132933

12. Ferrara LA, Raimondi AS, d’Episcopi L, Guida L, Dello Russo A, Marotta T. Olive oil and reduced need for antihypertensive medications. Arch Intern Med. 2000;160:837-42.

13. Ros E, Nunez I, Perez-Heras A, et al. A walnut diet improves endothelial function in hypercholesterolemic subjects: a randomized crossover trial. Circulation. 2004;109(13):1609-14

14. Thies F, Garry JMC, Yaqoob P, et al. Association of n-3 polyunsaturated fatty acids with stability of atherosclerotic plaques: a randomised controlled trial. Lancet. 2003;361: 477-85.

15. Sabate J, Haddad E, Tanzman JS, Jambazian $P$, Rajaram S. Serum lipid response to the graduated enrichment of a Step I diet with almonds: a randomized feedng trial. Am J Clin Nutr. 2003;77:1379-84.

16. Kris-Etherton PM, Harris WS, Appel LJ. Omega-3 fatty acids and cardiovascular disease: new recommendations from the American Heart Association. Arterioscler Thromb Vasc Biol. 2003;23(2):151-2.

17. Urquiaga I, Leighton F. Wine and health: evidence and mechanisms. World Rev Nutr Diet. 2005;95:122-39

18. North BJ, Sinclair DA. Sirtuins: A conserved key unlocking AceCS activity. Trends Biochem Sci. 2007:32(1):1-4.

19. Wood JG, Rogina B, Lavu S, et al. Sirtuin activators mimic caloric restriction and delay ageing in metazoans. Nature. 2004:430(7000): 686-9.

20. Naska A Oikonomou E, Trichopoulou A Psaltopoulou T, Trichopoulos D. Siesta in Healthy adults and coronary mortality in the general population. Arch Intern Med. 2007; 167:296-301.

21. David M. Eating from the tree: Nutrition lessons for the scientific soul. Altern Ther Health Med. 2004;10(6):10-3, 78-81.

22. de Lorgeril M, Renaud S, Mamelle N, et al Mediterranean alpha-linolenic acid-rich diet in secondary prevention of coronary heart disease. Lancet. 1994;343(8911):1454-9.

23. de Lorgeril M, Salen P, Martin JL, Monjaud I, Delaye J, Mamelle N. Mediterranean diet, traditional risk factors, and the rate of cardiovascular complications after myocardial infarction: final report of the Lyon Diet Heart Study. Circulation. 1999;99(6):779-85.

24. Singh RB, Dubnov G, Niaz MA, et al. Effect of an Indo-Mediterranean diet on progression of coronary artery disease in high risk patients: a randomised single-blind trial. Lancet. 2002; 360(9344):1455-61.

25. Skoldstam L, Hagfors L, Johansson G. An experimental study of a Mediterranean diet intervention for patients with rheumatoid arthritis. Ann Rheum Dis. 2003;62(3):208-14.

26. Toobert DJ, Glasgow RE, Strycker LA, et al. Biologic and quality-of-life outcomes from the Mediterranean Lifestyle Program: a randomized clinical trial. Diabetes Care. 2003:26(8): 2288-93.
27. Esposito K, Marfella R, Ciotola M, et al. Effect of a mediterranean-style diet on endothelial dysfunction and markers of vascular inflammation in the metabolic syndrome: a randomized trial. Jama. 2004;292(12):1440-6.

28. Knoops KTB, de Groot LC, Kromhout D, et al. Mediterranean diet, lifestyle factors, and 10year mortality in elderly European Men and women. JAMA. 2004;292:1433-1439.

29. Jula A, Marniemi J, Huupponen R, Virtanen A, Rastas M, Rönnemaa T. Effetcs of diet and simvastatin on serum lipids, insulin, and antioxidants in hypercholesterolemic men. Jama. 2002;287:598-605

30. Liese AD, Roach AK, Sparks KC, Marquart L, D'Agostino RB, Jr., Mayer-Davis EJ. Wholegrain intake and insulin sensitivity: the Insulin Resistance Atherosclerosis Study. Am J Clin Nutr. 2003;78(5):965-71

31. Jensen MK, Koh-Banerjee P, Hu FB, et al. Intakes of whole grains, bran, and germ and the risk of coronary heart disease in men. Am J Clin Nutr. 2004;80(6):1492-9.

32. McKeown NM, Meigs JB, Liu S, Wilson PW, Jacques PF. Whole-grain intake is favorably associated with metabolic risk factors for type 2 diabetes and cardiovascular disease in the Framingham Offspring Study. Am J Clin Nutr. 2002;76(2):390-8.

33. Behall KM, Scholfield DJ, Hallfrisch J. Wholegrain diets reduce blood pressure in mildly hypercholesterolemic men and women. J Am Diet Assoc. 2006;106(9):1445-9.

34. Adzersen KH, Jess P, Freivogel KW, Gerhard I, Bastert G. Raw and cooked vegetables, fruits, selected micronutrients, and breast cancer risk: a case-control study in Germany. Nutr Cancer. 2003;46(2):131-7.

35. Larsson SC, Giovannucci E, Bergkvist L, Wolk A. Whole grain consumption and risk of colorectal cancer: a population-based cohort of 60,000 women. Br J Cancer. 2005;92(9): 1803-7.

36. Nenonen MT, Helve TA, Rauma AL, Hanninen OO. Uncooked, lactobacilli-rich, vegan food and rheumatoid arthritis. $\mathrm{Br} J$ Rheumatol. 1998;37(3):274-81

37. Peltonen $R$, Nenonen M, Helve T, Hanninen $\mathrm{O}$, Toivanen P, Eerola E. Faecal microbial flora and disease activity in rheumatoid arthritis during a vegan diet. Br J Rheumatol. 1997; 36(1):64-8

38. Miller ER, 3rd, Erlinger TP, Young DR, et al. Results of the Diet, Exercise, and Weight Loss Intervention Trial (DEW-IT). Hypertension. 2002;40(5):612-8.

39. Michalsen A, Bierhaus A, Nawroth PP, Dobos GJ. [Glycotoxins and cellular dysfunction. A new mechanism for understanding the preventive effects of lifestyle modifications]. Bundesgesundheitsblatt Gesundheitsforschung Gesundheitsschutz. 2006;49(8):773-9.

40. Negrean M, Stirban A, Stratmann B, et al. Effects of low- and high-advanced glycation endproduct meals on macro- and microvascular endothelial function and oxidative stress in patients with type 2 diabetes mellitus. Am J Clin Nutr. 2007;85(5):1236-43.

41. Stirban A, Negrean M, Stratmann B, et al. Benfotiamine prevents macro- and microvascular endothelial dysfunction and oxidative stress following a meal rich in advanced glycation end products in individuals with type 2 diabetes. Diabetes Care. 2006;29(9):2064-71.

42. Ornish D, Brown SE, Scherwitz LW, et al. Can lifestyle changes reverse coronary heart disease? The Lifestyle Heart Trial. Lancet. 1990; 336(8708):129-33.

43. Ornish D, Scherwitz LW, Billings JH, et al. Intensive lifestyle changes for reversal of coronary heart disease. JAMA. 1998;280(23): 2001-7.
44. Ornish D, Weidner G, Fair WR, et al. Intensive lifestyle changes may affect the progression of prostate cancer. J Urol. 2005; 174(3):1065-9; discussion 1069-70.

45. Appel LJ, Champagne CM, Harsha DW, et al. Effects of comprehensive lifestyle modification on blood pressure control: main results of the PREMIER clinical trial. Jama. 2003; 289(16):2083-93.

46. Appel LJ, Moore TJ, Obarzanek E, et al. A clinical trial of the effects of dietary patterns on blood pressure. DASH Collaborative Research Group. N Engl J Med. 1997; 336(16):1117-24

47. Fields JZ, Walton KG, Schneider $\mathrm{RH}$, et al. Effect of a multimodality natural medicine program on carotid atherosclerosis in olde subjects: a pilot trial of Maharishi Vedic Medicine. Am J Cardiol. 2002;89(8):952-8.

48. Michalsen A, Pithan C. Ernährungstherapie. In: Dobos GJ, Deuse U, Michalsen A, eds. Chronische Erkrankungen Integrativ. Stuttgart: Elsevier; 2006

49. Hoffmann B, Moebus S, Michalsen A, et al. [Health-related control belief and quality of life in chronically ill patients after a behavioral intervention in an integrative medicine clinican observational study]. Forsch Komplementärmed Klass Naturheilkd. 2004;11(3):159-70.

50. Michalsen A, Knoblauch NT, Lehmann N, et al. Effects of lifestyle modification on the progression of coronary atherosclerosis, autonomic function, and angina--the role of GNB3 C825T polymorphism. Am Heart J. 2006 151(4):870-7.

51. Dansinger ML, Gleason JA, Griffith JL, Selker HP, Schaefer EJ. Comparison of the Atkins, Ornish, Weight Watchers, and Zone diets for weight loss and heart disease risk reduction: a randomized trial. Jama. 2005;293(1):43-53

52. Buchinger A. Fasting. In: Nowey D, ed. Clinician's Complete Reference to Complementary and Alternative Medicine. St. Louis: Mosby; 2000.

53. Buchinger O. Das Heilfasten und seine Hilfsmethoden Stuttgart: Hippokrates; 1932.

54. Wilhelmi de Toledo F. Therapeutisches Fasten nach Buchinger und Immunsystem: Erfahrungen und Hypothesen. Ärztezeit schrift f. Naturheilverf. 1995;36(5):331-341.

55. Ärztegesellschaft Heilfasten und Ernährung: Wilhelmi de Toledo F et al. Leitlinien zur Fas tentherapie. Forsch Kompl. 2002;9:189-198.

56. Wilhelmi de Toledo F. Buchinger Heilfasten: Ein Erlebnis für Körper und Geist Stuttgart: Trias Verlag; 2003

57. Brubacher D, Jordan P, Wilhelmi de Toledo F, Brubacher G. Predicition of weight development on a $250 \mathrm{kcal} /$ day diet by a simple two compartment model. Aktuelle Ernährungs medizin. 1998;6:293-98.

58. Schubmann R, Graban I, Hölz G, Zwingmann C. Ergebnisqualität stationärer Rehabilitation bei Patienten mit Adipositas. Deutsche Rentenversicherung. 1997:9-10:1-22.

59. Wilhelmi de Toledo F, Friebe $R$, Hebisch D Kuhn C, Platzer G, Scharg S. The Klinik Buchinger Programme for the treatment of obesity. In: Ditschuneit H, Gries FA, Haunte H, Schusziarra V, Wechsler JG, eds. Obesity in Europe 1993. London: Libbey; 1994:289-93.

60. Kjeldsen-Kragh J, Haugen M, Borchgrevink $\mathrm{CF}$, et al. Controlled trial of fasting and oneyear vegetarian diet in rheumatoid arthritis. Lancet. 1991;338:899-902.

61. Müller H, Wilhelmi de Toledo F, Resch KL. A systematic review of clinical studies on fasting and vegetarian diets in the treatment of rheumatoid arthritis. Scand. J. Rheumatol. 2000;30:1-10. 
62. Lord GM, Matarese G, Howard JK, Baker RJ, Bloom SR, Lechler RI. Leptin modulates the T-cell immune response and reverses starvation-induced immunosuppression. Nature. 1998;394(6696):897-901.

63. Fraser DA, Thoen J, Selvaag AM, Djoseland $\mathrm{O}$, Forre $\mathrm{O}$, Kjeldsen-Kragh J. A preliminary study of circadian serum cortisol concentrations in response to a 72-hour fast in rheumatoid arthritis patients not previously treated with corticosteroids. Clin. Rheumatol. 2001; 20:85-87

64. Hentges DJ. Does diet influence human fecal microflora composition? Nutr Rev. 1980; 38(10):329-36

65. Kjeldsen-Kragh J, Rashid T, Dybwad A, et al. Decrease in anti-Proteus mirabilis but not anti-Escherichia coli antibody levels in rheumatoid arthritis patients treated with fasting and a one year vegetarian diet. Ann Rheum Dis. 1995;54(3):221-4.

66. Michalsen A, Hoffmann B, Moebus S, Backer $M$, Langhorst J, Dobos GJ. Incorporation of fasting therapy in an integrative medicine ward: evaluation of outcome, safety, and effects on lifestyle adherence in a large prospective cohort study. J Altern Complement Med. 2005;11(4):601-7.

67. Hargraves WA, Hentall ID. Analgesic effects of dietary caloric restriction in adult mice. Pain. 2005;114(3):455-61.

68. Lipecki R. Klinische Studie zur Effizienz einer kombinierten Heilfastenbehandlung als Migränetherapie. Inaugural Dissertation. 1990; Universität Würzburg:1-53.

69. Huether G, Zhou D, Schmidt S, Wiltfang J, Ruther $\mathrm{E}$. Long-term food restriction downregulates the density of serotonin transporters in the rat frontal cortex. Biol Psychiatry. 1997;41(12):1174-80.

70. Michalsen A, Kuhlmann MK, Ludtke R, Backer M, Langhorst J, Dobos GJ. Prolonged fasting in patients with chronic pain syndromes leads to late mood-enhancement not related to weight loss and fasting-induced leptin depletion. Nutr Neurosci. 2006;9(5-6): 195-200.

71. Michalsen A, Schlegel F, Rodenbeck A, et al. Effects of short-term modified fasting on sleep patterns and daytime vigilance in nonobese subjects: results of a pilot study. Ann Nutr Metab. 2003;47(5):194-200.

72. Bergendahl $\mathrm{M}$, Vance $\mathrm{ML}$, Iranmanesh $\mathrm{A}$, Thorner MO, Veldhuis JD. Fasting as a metabolic stress paradigm selectively amplifies cortisol secretory burst mass and delays the time of maximal nycthemeral cortisol concentrations in healthy men. J Clin Endocrin Metab. 1996;81:692-99

73. Goschke H, Girard J, Stahl M. Der Stoffwechsel beim vollständigem Fasten. Unterschiedliches Verhalten bei Männern und Frauen sowie bei Normalpersonen und Adipösen. Klin Wschr. 1976:54:527-533.

74. Michalsen A, Schneider S, Rodenbeck A Ludtke R, Huether G, Dobos GJ. The shortterm effects of fasting on the neuroendocrine system in patients with chronic pain syndromes. Nutr Neurosci. 2003;6(1):11-8.

75. Schwartz MW, Seeley RJ. Seminars in medicine of the Beth Israel Deaconess Medical Center. Neuroendocrine responses to starvation and weight loss. N Engl J Med. 1997; 336(25):1802-11.

76. Goehler L, Hahnemann T, Michael N, et al. Reduction of plasma catecholamines in humans during clinically controlled severe underfeeding. Prev Med. 2000;30(2):95-102.

77. Dessi-Fulgheri P, Sarzani R, Serenelli M, Tamburrini P, Spagnalo D. Low Calorie Diet Enhances Renal, Hemodynamic and Humoral
Effects of Exogenous Atrial Peptide in Obese Hypertensives. Hypertension. 1999;33:658-662.

78. Goldhamer AC, Lisle DJ, Sultana $P$, et al. Medically supervised water-only fasting in the treatment of borderline hypertension. J Altern Complement Med. 2002;8(5):643-50.

79. Müller $H$, Wilhelmi de Toledo $F$, Schuck $P$ Resch KL. Blutdrucksenkung durch Fasten bei adipösen und nichtadipösen Hypertonikern. Perfusion. 2001;14:108-112.

80. Maoz E, Shamiss A, Peleg E, Salzberg M, Rosenthal $\mathrm{T}$. The role of atrial natriuretic peptide in natriuresis of fasting. J Hypertens. 1992;10(9):1041-4

81. Mattson MP, Wan R. Beneficial effects of intermittent fasting and caloric restriction on the cardiovascular and cerebrovascular systems. J Nutr Biochem. 2005;16(3):129-37.

82. Holloszy JO. Mortality rate and longevity of food-restricted exercising male rats: a reevaluation. J Appl Physiol. 1997;82(2):399-403.

83. Weindruch R. Caloric restriction, gene expression, and aging. Alzheimer Dis Assoc Disord. 2003;17 Suppl 2:S58-9.

84. Mattson MP. Energy Intake, Meal Frequency, and Health: A Neurobiological Perspective. Annu Rev Nutr. 2004

85. Mattson MP. The need for controlled studies of the effects of meal frequency on health. Lancet. 2005;365(9475):1978-80.

86. Heilbronn LK, de Jonge L, Frisard MI, et al. Effect of 6-month calorie restriction on biomarkers of longevity, metabolic adaptation, and oxidative stress in overweight individuals: a randomized controlled trial. Jama. 2006;295(13):1539-48.

87. Mattson MP. Energy intake, meal frequency, and health: a neurobiological perspective. Annu Rev Nutr. 2005;25:237-60

88. Bergendahl M, Evans WS, Pastor $C$, Patel A, Iranmanesh A, Veldhuis JD. Short-term fasting suppresses leptin and (conversly) activates disorderly growth hormone secretion in midluteal phase woman - a clinical research center study. J Clin Endocrin Metab. 1999; 84:883-894.

89. Göschke H, Girard J, Stahl M. Der Stoffwechsel bei vollständigem Fasten. Unterschiedliches Verhalten bei Männern und Frauen sowie bei Normalpersonen und Adipösen. Klin Wschr. 1976;54:527-533.

90. Martin B, Mattson MP, Maudsley S. Caloric restriction and intermittent fasting: two potential diets for successful brain aging. Ageing Res Rev. 2006;5(3):332-53.

91. Sell DR, Lane MA, Johnson WA, et al. Longevity and the genetic determination of collagen glycoxidation kinetics in mammalian senescence. Proc Natl Acad Sci U S A. 1996; 93(1):485-90

92. Cefalu WT, Bell-Farrow AD, Wang ZQ, et al. Caloric restriction decreases age-dependent accumulation of the glycoxidation products, $\mathrm{N}$ epsilon-(carboxymethyl)lysine and pentosidine, in rat skin collagen. J Gerontol. 1995; 50:B337-341

93. Aljada A, Mohanty P, Ghanim $H$, et al. Increase in intranuclear nuclear factor kappaB and decrease in inhibitor kappaB in mononuclear cells after a mixed meal: evidence for a proinflammatory effect. Am J Clin Nutr. 2004; 79(4):682-90

94. Schiekofer S, Franke S, Andrassy M, et al. Postprandial mononuclear NF-kB activation is independent of the AGE-content of a single meal. Experimental and Clinical Endocrinology \& Diabetes. 2006: in press.

95. Exton MS. Infection-induced anorexia: active host defence strategy. Appetite. 1997;29(3): 369-83.
96. Owen OE, Smalley KJ, D'Alessio DA, Mozzoli MA, Dawson EK. Protein, fat, and carbohydrate requirements during starvation: anaplerosis and cataplerosis]. Am J Clin Nutr. 1998;68(1):12-34

97. Wechsler J, al. e. Ergebnisse der Adipositasbehandlung mit angereicherter Molke. 1986; 11:71-79.

98. Norrelund $H$, Nair KS, Jorgensen JO, Christiansen JS, Moller N. The protein-retaining effects of growth hormone during fasting involve inhibition of muscle-protein breakdown. Diabetes. 2001;50(1):96-104.

99. Lützner H. Fasten/ Fastentherapie: Grundlagen und Methodik. In: Bühring M, Kemper $\mathrm{FH}$, Matthiessen PF, eds. Naturheilverfahren und Unkonventionelle Medizinische Richtungen. Berlin: Springer LoseblattSysteme; 1998:1-26.

100.Peper E. Evaluation der Effekte und Erfolge von stationären Heilfastenmassnahmen Frankfurt/M., Berlin, Bern, Bruxelles, New York, Wien: Peter Lang - Europäischer Verlag der Wissenschaften; 1999

\section{Korrespondenzadresse:}

PD Dr. med. Andreas Michalsen

Kliniken Essen-Mitte

Abteilung Innere Medizin V,

Integrative Medizin und Naurheilkunde

Am Deimelsberg 34a, DE-45276 Essen

andreas.michalsen@uni-duisburg-essen.de 